\title{
Particle Dynamics around Weakly Magnetized Reissner-Nordström Black Hole
}

\author{
Bushra Majeed, ${ }^{1}$ Mubasher Jamil, ${ }^{1}$ and Saqib Hussain ${ }^{2}$ \\ ${ }^{1}$ Department of Mathematics, School of Natural Sciences (SNS), National University of Sciences and Technology (NUST), \\ H-12, Islamabad 44000, Pakistan \\ ${ }^{2}$ Department of Physics, School of Natural Sciences (SNS), National University of Sciences and Technology (NUST), \\ H-12, Islamabad 44000, Pakistan
}

Correspondence should be addressed to Bushra Majeed; bushra.majeed@sns.nust.edu.pk

Received 7 September 2015; Revised 24 November 2015; Accepted 25 November 2015

Academic Editor: Juan José Sanz-Cillero

Copyright (C) 2015 Bushra Majeed et al. This is an open access article distributed under the Creative Commons Attribution License, which permits unrestricted use, distribution, and reproduction in any medium, provided the original work is properly cited. The publication of this article was funded by SCOAP $^{3}$.

Considering the geometry of Reissner-Nordström (RN) black hole immersed in magnetic field, we have studied the dynamics of neutral and charged particles. A collision of particles in the inner stable circular orbit is considered and the conditions for the escape of colliding particles from the vicinity of black hole are given. The trajectories of the escaping particle are discussed. Also, the velocity required for this escape is calculated. It is observed that there is more than one stable region if magnetic field is present in the accretion disk of black hole, so the stability of ISCO increases in the presence of magnetic field. Effect of magnetic field on the angular motion of neutral and charged particles is observed graphically.

\section{Introduction}

The geometrical structure of the spacetime around black hole (BH) could be understood better by studying the dynamics of particles in the vicinity of black holes $[1,2]$. Circular geodesics give information about geometry of spacetime $[3,4]$. The motion of test particles helps to study the gravitational fields of objects experimentally and to compare the observations with the predictions about observable effects (light like deflection, gravitational time delay, and perihelion shift). Presence of plasma is responsible for magnetic field [5], in the surroundings of the black hole [6,7]. Near the event, horizon effect of magnetic field is strong but not enough to disturb the geometry of the black hole. Motion of a charged particle around black hole in the presence of magnetic field gets influenced $[8,9]$. Black holes with such scenario are known as "weakly magnetized" [10]; that is, the magnetic field strength lies within $B \sim 10^{4}-10^{8} \ll$ $10^{19}$ Gauss. Magnetic field is responsible for transferring energy to the particles moving in the geometry around black hole, so that their escape to spatial infinity is possible [11]. Hence, the collision of charged particles near the black hole may produce much higher energy in the presence of magnetic field than in its absence. In [12-15], the effects of magnetic fields on the charged particles around black holes were investigated. Timelike geodesics in modified gravity black hole in the presence of axially symmetric magnetic field are studied in [16]. In [17], authors have studied the dynamics of a charged particle around a weakly magnetized naked singularity, in the Janis-Newman-Winicour (JNW) spacetime. Kaya [18] studied the motion of charged particles around a five-dimensional rotating black hole in a uniform magnetic field and found stable circular orbits around the black hole. In literature, many aspects of the particles motion in the vicinity of RN-black hole have been studied. In [19], authors have studied the high energy collisions phenomenon between the particles, currently termed as BSW mechanism. In [20], the spatial regions for circular motion of neutral and charged test particles around RN-BH and naked singularities have been studied. Critical escape velocity for a charged particle moving around a weakly magnetized Schwarzschild black hole has been studied in [21]. We consider Reissner-Nordström (RN) black hole is surrounded by an axially symmetric magnetic field which is homogeneous at infinity. Particles in the accretion 
disc move in circular orbits in the equatorial plane. Following the work done by Al Zahrani et al. [21], collision of a neutral and a charged particle with another neutral particle is studied in the vicinity of magnetized $\mathrm{RN}-\mathrm{BH}$. We focus on under what circumstances the particle can escape to infinity after collision. To evade the complication in modeling the particle's motion around a black hole under the influence of both gravitational and magnetic forces, we first consider the motion of a neutral particle in the absence of magnetic field. The study of particle dynamics around RN spacetime is also relevant as this metric represents the extreme $\mathrm{RN}-\mathrm{BH}$ and a naked singularity as special cases.

Outline of the paper is as follows. In Section 2, metric of $\mathrm{RN}-\mathrm{BH}$ is discussed and escape velocity of a neutral particle is calculated. In Section 3, the equations of motion of a charged particle moving around weakly magnetized $\mathrm{RN}-\mathrm{BH}$ are derived. Trajectories of the particles moving around the extremal RN-BH are plotted in Section 4. In Section 5, the dimensionless form of the equations is given. In Section 6, trajectories for escape energy and escape velocity of the particle are presented. Motion of the particle is initially considered in the equatorial plane for the sake of simplicity. The metric signature is $(-,+,+,+)$ and $c=1, G=1$.

\section{Escape Velocity for a Neutral Particle}

We first work for the escape velocity of a neutral particle in the absence of magnetic field. The RN-BH metric is given by

$$
d s^{2}=-f(r) d t^{2}+\frac{1}{f(r)} d r^{2}+r^{2}\left(d \theta^{2}+\sin ^{2} \theta d \phi^{2}\right),
$$

where

$$
f(r)=1-\frac{2 M}{r}+\frac{Q^{2}}{r^{2}}
$$

Here, $M$ is the mass and $Q$ is electric charge of the black hole. Horizon of the RN-BH is located at

$$
r_{ \pm}:=M \pm \sqrt{M^{2}-Q^{2}}
$$

If $M>Q$, there are two real positive roots; the larger root corresponds to the event horizon and the smaller root refers to the Cauchy horizon which is associated with the timelike singularity at $r=0$. Black hole is known to be extremal black hole if $M=Q$ and it has only one event horizon at $r=M=Q$. If $M<Q$, there is no real root of the equation $f(r)=0$ and there is no event horizon. This case is known as naked singularity of RN spacetime. Symmetries of the black hole metric are along the time translation and rotation around symmetry axis. The corresponding constants of motion can be calculated using the Killing vectors:

$$
\begin{aligned}
& \xi_{(t)}^{\mu} \partial_{\mu}=\partial_{t}, \\
& \xi_{(\phi)}^{\mu} \partial_{\mu}=\partial_{\phi} .
\end{aligned}
$$

Here, $\xi_{(t)}^{\mu}=(1,0,0,0)$ and $\xi_{(\phi)}^{\mu}=(0,0,0,1)$. The corresponding conserved quantities are the total energy $\mathscr{E}$ of the moving particle and its azimuthal angular momentum $L_{z}$ :

$$
\begin{gathered}
\dot{t}=\frac{\mathscr{E}}{f}, \\
\dot{\phi}=\frac{L_{z}}{r^{2}} .
\end{gathered}
$$

Over dot is the differentiation with respect to proper time $\tau$ and $f:=f(r)$. From the normalization condition $u^{\mu} u_{\mu}=-1$, we have

$$
\begin{aligned}
\dot{r}^{2} & =\mathscr{E}^{2}-U_{\mathrm{eff}}, \\
U_{\mathrm{eff}} & =\left(1-\frac{2 M}{r}+\frac{Q^{2}}{r^{2}}\right)\left(1+\frac{L_{z}^{2}}{r^{2}}\right) .
\end{aligned}
$$

We considered $\theta=\pi / 2$, that is, the planar motion of the particle. Solving $d U_{\text {eff }} / d r=0$, we get the value of $r$ corresponding to the extreme values of the effective potential (the convolution point) [22]:

$$
r_{ \pm}=\frac{L_{z}^{2} \pm \sqrt{L_{z}^{4}-L_{z}^{2} 8 M^{2}}}{2 M} .
$$

The ISCO is at $r=4 M$ for extreme RN-BH $(Q=M)$. For $Q=$ 0 , it reduces to $r=6 M$ (Schwarzschild black hole) [21]. The corresponding energy and the azimuthal angular momentum of the particle (in ISCO) are, respectively,

$$
\begin{aligned}
\mathscr{E}_{o} & =\frac{\left(Q^{2}+r_{o}\left(-2 M+r_{o}\right)\right)^{2}}{r_{o}^{2}\left(2 Q^{2}+r_{o}\left(-3 M+r_{o}\right)\right)}, \\
L_{z o} & =\frac{\sqrt{Q^{2} r_{o}^{2}-M r_{o}^{3}}}{\sqrt{-2 Q^{2}+3 M r_{o}-r_{o}^{2}}} .
\end{aligned}
$$

For extremal black hole case, that is, at $Q=M,(8)$ becomes

$$
\begin{aligned}
& \mathscr{E}_{o}^{(e)}=\frac{\left(M^{2}+r_{o}\left(-2 M+r_{o}\right)\right)^{2}}{r_{o}^{2}\left(2 M^{2}+r\left(-3 M+r_{o}\right)\right)}, \\
& L_{z o}^{(e)}=\frac{\sqrt{M\left(M-r_{o}\right) r_{o}^{2}}}{\sqrt{-2 M^{2}+3 M r_{o}-r_{o}^{2}}} .
\end{aligned}
$$

Now, consider the collision of a particle, moving in the ISCO, with another particle which is coming from infinity (initially at rest). This collision may result in three possibilities (depending on the progression of the collision): (i) a bounded motion, (ii) particle captured by black hole, and (iii) particle escape to infinity. Orbit of the particle alters slightly if the energy and angular momentum of the particle do not undergo a major change; otherwise, the particle can move away from the original path resulting in captured by black hole or an escape to infinity may occur. Collision of the particles changes the equatorial plane of the moving particle, but 
since the metric is spherical symmetric, all the equatorial planes are similar. We consider the collision occurring in such a way that (i) the azimuthal angular momentum remains invariant and (ii) initial radial velocity also remains the same. Hence, only the change in energy will be considered for determining the motion of particle after collision. These conditions are imposed for simplification only. Particle gains an escape velocity $\left(v_{\text {esc }}\right)=v_{\perp}$ in orthogonal direction of the equatorial plane after collision [23] and its momentum and energy (in the new equatorial plan) become

$$
L^{2}=r_{o}^{2} v_{\mathrm{esc}}^{2}+L_{z o}^{2}
$$

Here, $v_{\text {esc }} \equiv-r \dot{\theta}_{o}$ and $\dot{\theta}_{o}$ denotes the particles' initial polar angular velocity. Energy of the particle is

$$
\begin{aligned}
& \mathscr{E}_{\text {new }}=\sqrt{\left(1+\frac{Q^{2}}{r_{o}^{2}}-\frac{2 M}{r_{o}}\right) v_{\mathrm{esc}}^{2}+\mathscr{E}_{o}^{2},} \\
& \mathscr{E}_{\text {new }}^{(e)}=\sqrt{\frac{\left(M-r_{o}\right)^{2} v_{\mathrm{esc}}^{2}}{r_{o}^{2}}+\mathscr{E}_{o}^{2},}
\end{aligned}
$$

with $\mathscr{E}_{o}$, given in (9). After collision, particle gains greater angular momentum and energy as compared to that before collision. From (11), it is clear that, in the asymptotic limit $(r \rightarrow \infty), \mathscr{E}_{\text {new }} \rightarrow \mathscr{E}_{o}=1$. So for unbounded motion (escape), particle requires $\mathscr{E} \equiv \mathscr{E}_{\text {new }} \geq 1$. Hence, for escape to infinity, the necessary condition is

$$
\begin{aligned}
& v_{\mathrm{esc}} \geq \frac{r_{o} \sqrt{1-\mathscr{E}_{o}}}{\sqrt{-Q^{2}+2 M r_{o}-r^{2}}}, \\
& v_{\mathrm{esc}}^{(e)} \geq \frac{r_{o} \sqrt{1-\mathscr{E}_{o}}}{\sqrt{-M^{2}+2 M r_{o}-r_{o}^{2}}} .
\end{aligned}
$$

We have solved (11) taking $\mathscr{E}_{\text {new }} \geq 1$ and the quantities with subscript $e$ denote the extremal black hole case.

\section{Charged Particle around RN-BH Surrounded by Magnetic Field}

The presence of magnetic field interrupts the motion of a charged particle around black hole. To know the aftermath of this perturbation, let us start with the Lagrangian of the moving particle as

$$
\mathscr{L}=\frac{1}{2} g_{\mu \nu} \dot{x}^{\mu} \dot{x}^{\nu}+\frac{q}{m} A_{\mu} \dot{x}^{\mu} .
$$

Here, $m$ is mass of the particle and $q$ is the charge of particle. The Killing vector equation $\square \xi^{\mu}=0$ resembles the Maxwell equation for $A^{\mu}$ in the Lorentz gauge $A_{; \mu}^{\mu}=0[24,25]$; here, $\xi^{\mu}$ denotes the Killing vector and $A^{\mu}$ is the 4-potential defined as $[24,25]$

$$
A^{\mu}=\frac{\mathscr{B}}{2} \xi_{(\phi)}^{\mu}-\frac{q}{2 m} \xi_{(t)}^{\mu}
$$

with $\mathscr{B}$ as the magnetic field strength given as

$$
\mathscr{B}^{\mu}=-\frac{1}{2} e^{\mu \nu \lambda \sigma} F_{\lambda \sigma} u_{\nu},
$$

and using Levi Civita symbol, $\epsilon^{\mu \nu \lambda \sigma}$, one can write

$$
e^{\mu \nu \lambda \sigma}=\frac{\epsilon^{\mu \nu \lambda \sigma}}{\sqrt{-g}}, \quad \epsilon_{0123}=1, g=\operatorname{det}\left(g_{\mu \nu}\right) .
$$

The Maxwell tensor, $F_{\mu \nu}$, is defined as

$$
F_{\mu \nu}=A_{\nu, \mu}-A_{\mu, \nu}=A_{\nu ; \mu}-A_{\mu ; \nu}
$$

For a local observer in RN geometry, $u_{0}^{\mu}=(1 / \sqrt{f}) \xi_{(t)}^{\mu}$. The only two components of $F_{\mu \nu}$ will survive which are $F_{10}=-F_{01}$ and $F_{13}=-F_{31}$.

Using the Euler-Lagrange equations for the Lagrangian defined in (13), one can get easily

$$
\begin{aligned}
\dot{t} & =\frac{\mathscr{E}}{f(r)}, \\
\dot{\phi} & =\frac{L_{z}}{r^{2} \sin ^{2} \theta}-B .
\end{aligned}
$$

Here, $B \equiv q \mathscr{B} / 2 m$. The normalization condition $\left(u^{\mu} u_{\mu}=-1\right)$ gives

$$
\begin{aligned}
\mathscr{E}^{2} & =\dot{r}^{2}+r^{2} f \dot{\theta}^{2}-U_{\mathrm{eff}} \\
U_{\mathrm{eff}} & =f\left[1+r^{2} \sin ^{2} \theta\left(\frac{L_{z}}{r^{2} \sin ^{2} \theta}-B\right)^{2}\right] .
\end{aligned}
$$

Equation of motion of a charged particle moving in an external electromagnetic field $F_{\mu \nu}$ satisfies

$$
\ddot{x}^{\mu}+\Gamma_{\gamma \sigma}^{\mu} \dot{x}^{\nu} \dot{x}^{\sigma}=\frac{q}{m} F_{\alpha}^{\mu} \dot{x}^{\alpha}
$$

Using (20) for the metric defined in (1), we get the dynamical equations for $\theta$ and $r$ given as

$$
\begin{aligned}
\ddot{\theta}= & \frac{-2}{r} \dot{r} \dot{\theta}+\frac{L_{z}^{2} \cos \theta}{r^{4} \sin ^{3} \theta}-B^{2} \sin \theta \cos \theta, \\
\ddot{r}= & \dot{\theta}^{2}\left(\frac{2 r^{2}-3 r r_{g}+4 Q^{2}}{2 r}\right)-\frac{r_{g}}{2 r^{2}}+\frac{Q^{2}}{r^{3}} \\
& +\frac{L_{z}^{2}}{2 r^{2} \sin ^{2} \theta}\left(\frac{2 r^{2}-3 r r_{g}+4 Q^{2}}{r^{3}}\right) \\
& -B^{2} \sin ^{2} \theta\left(\frac{2 r^{2}-r r_{g}}{2 r}\right)+B L_{z}\left(\frac{r r_{g}-2 Q^{2}}{r^{3}}\right) \\
& +\frac{q^{2} \mathscr{E}}{2 m^{2}}\left(\frac{r r_{g}-2 Q^{2}}{r^{3}}\right) .
\end{aligned}
$$

Here, $r_{g}=2 M$. 


\section{Dynamical Equations in Dimensionless Form}

For the sake of convenience, we can rewrite the dynamical equations of $r$ and $\theta$ in dimensionless form by introducing the following dimensionless quantities [23]:

$$
\begin{aligned}
\sigma & =\frac{\tau}{r_{g}}, \\
\rho & =\frac{r}{r_{g}}, \\
\ell & =\frac{L_{z}}{r_{g}}, \\
b & =B r_{g}, \\
\widetilde{q} & =\frac{Q}{r_{g}}, \\
q^{\prime} & =\frac{q}{r_{g}}, \\
m^{\prime} & =\frac{m_{g}}{r_{g}} .
\end{aligned}
$$

Here, $r_{g}=2 M$. Using the quantities defined in (22), (18), and (21), take the following forms:

$$
\begin{aligned}
& \frac{d \phi}{d \sigma}=\frac{\left(\ell-b \rho^{2} \sin ^{2} \theta\right)}{\rho^{2} \sin ^{2} \theta} \\
& \frac{d^{2} \theta}{d \sigma^{2}}=\frac{-2}{\rho} \frac{d \rho}{d \sigma} \frac{d \theta}{d \sigma}+\frac{\ell^{2} \cos \theta}{\rho^{4} \sin ^{3} \theta}-b^{2} \sin \theta \cos \theta, \\
& \frac{d^{2} \rho}{d \sigma^{2}}=\frac{1}{2 m^{\prime 2} \rho^{5}}\left(\mathscr{E} \rho^{2} q^{\prime 2}\left(-2 \widetilde{q}^{2}+\rho\right)\right)+\frac{1}{2 \rho^{5}}\left(2 \widetilde{q}^{2} \rho^{2}\right. \\
& \quad+4 b \ell \widetilde{q}^{2} \rho^{2}-\rho^{3}+2 b \ell \rho^{3}+\frac{4 \ell^{2} \widetilde{q}^{2}-3 \ell^{2}+\rho 2 \ell^{2} \rho}{\sin \theta} \\
& \quad+b^{2}(1-2 \rho) \rho^{5} \sin ^{2} \theta \\
& \left.\quad+\left(\frac{d \theta}{d \sigma}\right)^{2} \rho^{4}\left(4 \widetilde{q}^{2}+\rho(-3+2 \rho)\right)\right) .
\end{aligned}
$$

For extremal black hole case, it becomes

$$
\begin{aligned}
& \frac{d^{2} \rho}{d \sigma^{2}}=\frac{-1}{4 \rho^{5}}\left[( - 1 + 2 \rho ) \left(\rho^{2}-2 b \ell \rho^{2}+\frac{2 \ell^{2}-2 \ell^{2} \rho}{\sin \theta}\right.\right. \\
& \left.\left.+2 b^{2} \rho^{5} \sin ^{2} \theta-2\left(\frac{d \theta}{d \sigma}\right)^{2}(-1+\rho) \rho^{4}\right)\right] \\
& +\frac{\mathscr{E} \rho^{2} q^{\prime 2}}{4 \rho^{5} m^{\prime 2}}(-1+2 \rho)
\end{aligned}
$$

For the particle moving in equatorial plane, (25) and (26) become

$$
\begin{aligned}
& \frac{d^{2} \rho}{d \sigma^{2}}=\frac{1}{2 m^{\prime 2} \rho^{5}}\left(\mathscr{E} \rho^{2} q^{\prime 2}\left(-2 \widetilde{q}^{2}+\rho\right)\right)+\frac{1}{2 \rho^{5}}\left(2 \widetilde{q}^{2} \rho^{2}\right. \\
& \quad-4 b \ell \widetilde{q}^{2} \rho^{2}-\rho^{3}+2 b \ell \rho^{3}+4 \ell^{2} \widetilde{q}^{2}-3 \ell^{2} \rho+2 \ell^{2} \rho \\
& \left.\quad+b^{2}(1-2 \rho) \rho^{5}\right), \\
& \frac{d^{2} \rho}{d \sigma^{2}}=\frac{-1}{4 \rho^{5}}[(-1+2 \rho) \\
& \left.\quad \cdot\left(\rho^{2}-2 b \ell \rho^{2}+2 \ell^{2}-2 \ell^{2} \rho+2 b^{2} \rho^{5}\right)\right] \\
& +\frac{\mathscr{E} \rho^{2} q^{\prime 2}}{4 \rho^{5} m^{\prime 2}}(-1+2 \rho) .
\end{aligned}
$$

Using the built-in command NDSolve in Mathematica 8.0, (28) can be solved and the behavior of the obtained interpolating function can be better understood by plotting it against $\sigma$. Using (22) for (19), we obtain

$$
\mathscr{E}^{2}=\left(\frac{d \rho}{d \sigma}\right)^{2}+\rho^{2}\left(1+\frac{\tilde{q}^{2}}{\rho^{2}}-\frac{1}{\rho}\right)\left(\frac{d \theta}{d \sigma}\right)^{2}+U_{\mathrm{eff}},
$$

and for extreme black hole,

$$
\mathscr{E}^{2^{(e)}}=\left(\frac{d \rho}{d \sigma}\right)^{2}+\rho^{2}\left(\frac{2 \rho+1}{2 \rho}\right)^{2}\left(\frac{d \theta}{d \sigma}\right)^{2}+U_{\mathrm{eff}} .
$$

The effective potential given in (19) becomes

$$
U_{\mathrm{eff}}=\left(1+\frac{\tilde{q}^{2}}{\rho^{2}}-\frac{1}{\rho}\right)\left[1+\frac{\left(\ell-b \rho^{2} \sin ^{2} \theta\right)^{2}}{\rho^{2} \sin ^{2} \theta}\right],
$$

and for extreme black hole it becomes

$$
U_{\mathrm{eff}}^{(e)}=\left(\frac{2 \rho-1}{2 \rho}\right)^{2}\left[1+\frac{\left(\ell-b \rho^{2} \sin ^{2} \theta\right)^{2}}{\rho^{2} \sin ^{2} \theta}\right] .
$$

For the particle moving around $\mathrm{RN}-\mathrm{BH}$ in the equatorial plane, $\theta=\pi / 2$, at radius $\rho_{o}$, (30)-(32) become

$$
\begin{aligned}
\mathscr{E}_{o}^{2} & =\left(\frac{d \rho_{o}}{d \sigma}\right)^{2}+U_{\mathrm{eff}}, \\
U_{\mathrm{eff}} & =\left(1+\frac{\tilde{q}^{2}}{\rho_{o}^{2}}-\frac{1}{\rho_{o}}\right)\left[1+\frac{\left(\ell-b \rho_{o}^{2}\right)^{2}}{\rho_{o}^{2}}\right] .
\end{aligned}
$$

For extreme black hole,

$$
U_{\mathrm{eff}}^{(e)}=\left(\frac{2 \rho_{o}-1}{2 \rho_{o}}\right)^{2}\left[1+\frac{\left(\ell-b \rho_{o}^{2}\right)^{2}}{\rho_{o}^{2}}\right] .
$$

Again, considering the ideal scenario of collision which does not change the azimuthal angular momentum of 
the particle except its energy, that is, $\mathscr{E}_{o} \rightarrow \mathscr{E}$, defined as

$$
\mathscr{E}=\left[\mathscr{E}_{o}+\left(1+\frac{\tilde{q}^{2}}{\rho_{o}^{2}}-\frac{1}{\rho_{o}}\right) v_{\mathrm{esc}}^{2}\right]^{1 / 2}
$$

for extremal black hole,

$$
\mathscr{E}^{(e)}=\left[\mathscr{E}_{o}+\left(\frac{2 \rho_{o}-1}{2 \rho_{o}}\right)^{2} v_{\mathrm{esc}}^{2}\right]^{1 / 2}
$$

where $\mathscr{E}_{o}$ is the energy defined in (33). As already mentioned, when $\rho \rightarrow \infty$ the energy $\mathscr{E} \rightarrow 1$. So for the unbound motion the energy of the particle should be $\mathscr{E} \geq 1$. Solving (36) at $\mathscr{E}=1$, for escape velocity of the particle, we get the following expression:

$$
v_{\mathrm{esc}}=\sqrt{\frac{1-\left(1+\left(\ell / \rho_{o}^{2}-b\right)^{2}\right)^{2}\left(1+\tilde{q}^{2} / \rho_{o}^{2}-1 / \rho_{o}\right)^{2}}{1+\widetilde{q}^{2} / \rho_{o}^{2}-1 / \rho_{o}}},
$$

and for extremal RN-BH

$$
v_{\mathrm{esc}}^{2^{(e)}}=\frac{-\ell^{2}\left(1-2 \rho_{o}\right)^{2}+2 b \ell\left(1-2 \rho_{o}\right)^{2} \rho_{o}^{2}-\rho_{o}^{2}\left(1+\rho_{o}\left(-4+b^{2}\left(1-2 \rho_{o}\right)^{2} \rho_{o}\right)\right)}{\left(1-2 \rho_{o}\right)^{2} \rho_{o}^{2}} .
$$

For simplicity, we are considering the particle to be initially in ISCO; further, we discuss the behavior of the particle when it escapes to asymptotic infinity. The only parameters required for describing the motion of the particle are the parameters $\ell$ and $b$ defined in terms of $\rho_{o}$ and the energy of the particle. The expression for the parameters $\ell$ and $b$ in terms of $\rho$ could be obtained by dealing with (35). The first and second derivatives of the effective potential defined in (35) are

$$
\begin{aligned}
\frac{d}{d \rho} U_{\mathrm{eff}} & =\frac{\left(-1+2 \rho_{o}\right)\left(-2 \ell^{2}\left(-1+\rho_{o}\right)+\rho_{o}^{2}-2 b \ell \rho_{o}^{2}+2 b^{2} \rho_{o}^{5}\right)}{2 \rho_{o}^{5}} \\
\frac{d^{2}}{d \rho^{2}} U_{\mathrm{eff}} & =\frac{2 b \ell \rho_{o}^{2}\left(-3+4 \rho_{o}\right)+2 \ell^{2}\left(5+6\left(-2+\rho_{o}\right) \rho_{o}\right)+\rho_{o}^{2}\left(3-4 \rho_{o}+4 b^{2} \rho_{o}^{4}\right)}{2 \rho_{o}^{6}} .
\end{aligned}
$$

The values of $\ell$ and $b$ can be found by solving simultaneously $(d / d r) U_{\text {eff }}=0$ and $\left(d^{2} / d r^{2}\right) U_{\text {eff }}=0$, as given in the following:

$$
\begin{aligned}
& \ell= \pm \frac{\sqrt{-3 \rho_{o}^{5}\left(-2+\rho_{o}\right)^{3}} \sqrt{-\left(10 \rho_{o}^{3}-13 \rho_{o}^{4}+4 \rho_{o}^{5}+\sqrt{-3\left(-2+\rho_{o}\right)^{3} \rho_{o}^{5}}\right) /\left(1-2 \rho_{o}\right)^{2} \rho_{o}^{5}\left(-3+2 \rho_{o}\right)}}{2\left(-2+\rho_{o}\right)^{2}} \\
& b=\frac{1}{2} \sqrt{-\frac{10 \rho_{o}^{3}-13 \rho_{o}^{4}+4 \rho_{o}^{5}+\sqrt{-3\left(-2+\rho_{o}\right)^{3} \rho_{o}^{5}}}{\rho_{o}^{5}\left(-1+2 \rho_{o}\right)^{2}\left(2 \rho_{o}-3\right)}}
\end{aligned}
$$

In Section 7 , the parameters $\ell$ and $b$ are plotted against $\rho$.

\section{Effect of Magnetic Field on Motion of Particles}

Consider the neutral particle moving around RN-BH. Writing the equations associated with the constants of motion, in dimensionless form, we have

$$
\frac{d \rho}{d \sigma}= \pm \frac{\sqrt{\rho^{4} \mathscr{E}^{2}-\left(\rho^{2}-\rho+\widetilde{q}^{2}\right)\left(\rho^{2}+\ell^{2}\right)}}{\rho^{2}}
$$

where positive sign is for the particle going away from the black hole and negative sign is for the path of an ingoing particle; also

$$
\frac{d \phi}{d \sigma}=\frac{\ell}{\rho^{2}} .
$$


Using (42) and (43) together, we have

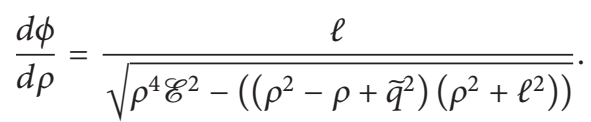

For extremal black hole, it becomes

$$
\frac{d \phi}{d \rho}=\frac{2 \ell}{\sqrt{4 \rho^{4} \mathscr{E}^{2}-\left((2 \rho-1)^{2}\left(\rho^{2}+\ell^{2}\right)\right)}} .
$$

It is observed graphically that a particle having less angular momentum approaches the black hole more closely as compared to the one having large angular momentum. This shows that when a particle does not move radially, its chances for approaching the black hole event horizon are very less (Figure 1). For a charged particle moving around RN-BH in the presence of magnetic field, we can write

$$
\begin{aligned}
& \frac{d \rho}{d \sigma}= \pm \frac{\sqrt{\mathscr{E}^{2} \rho^{4}-\left(\rho^{2}-\rho+\widetilde{q}^{2}\right)\left(\rho^{2}+\left(\ell-b \rho^{2}\right)^{2}\right)}}{\rho^{2}} \\
& \frac{d \phi}{d \sigma}=\frac{\left(\ell-b \rho^{2}\right)}{\rho^{2}} .
\end{aligned}
$$

Using (46) and (47) together, we have

$$
\frac{d \phi}{d \rho}= \pm \frac{\left(\ell-b \rho^{2}\right)}{\sqrt{\rho^{4} \mathscr{E}^{2}-\left(\rho^{2}-\rho+\widetilde{q}^{2}\right)\left(\rho^{2}+\left(\ell-b \rho^{2}\right)^{2}\right)}} .
$$

For extremal black hole,

$$
\frac{d \phi}{d \rho}= \pm \frac{2\left(\ell-b \rho^{2}\right)}{\sqrt{4 \rho^{4} \mathscr{E}^{2}-(2 \rho-1)^{2}\left(\rho^{2}+\left(\ell-b \rho^{2}\right)^{2}\right)}} .
$$

Change in $\phi$ during the motion of particle, around RN-BH, starting its motion from some finite distance is shown in Figure 2. It is observed that behavior of angular motion of particle is linked with the strength of magnetic field.

\section{Behavior of Effective Potential}

In this section, the behaviors of effective potentials of particle are studied graphically and the energy required for its escape to infinity or for bounded motion is discussed. In Figure 3, we have plotted the effective potential as a function of $\rho$. There are two minima $u_{\text {min }}$ and $u_{1 \text { min }}$, in the presence of magnetic field, while in the absence of magnetic field there is only one minimum, $u_{\min }$. Hence, we can say that the presence of magnetic field increases the possibility of the particle to move in a stable orbit. A comparison of effective potential of Schwarzschild black hole with that of $\mathrm{RN}-\mathrm{BH}$ is established in Figure 4. It is clear that the maxima for the effective potential of particle moving around $\mathrm{RN}-\mathrm{BH}$ have greater value in comparison with the maxima of effective potential for Schwarzschild black hole. Since a particle moving around black hole could be captured if it has energy greater than

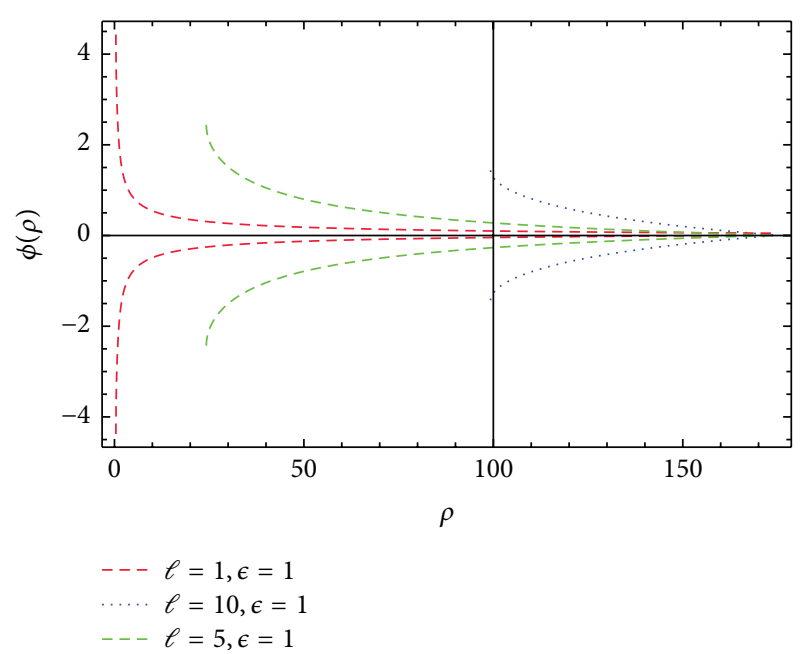

FIGURE 1: Behavior of angular motion linked with angular momentum of the particle.

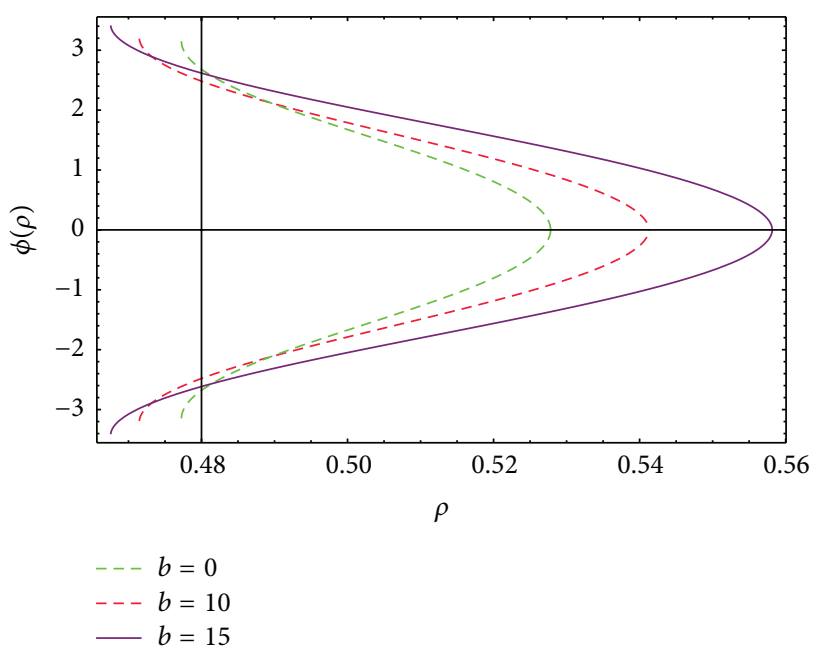

FIGURE 2: Effect of magnetic field on the charged moving particle, for $\mathscr{E}=1, \ell=10$.

maxima of its effective potential, it will move back to infinity or may reside in some stable orbit. Therefore, we can say that the possibility for a particle to escape from the vicinity of $\mathrm{RN}-\mathrm{BH}$ or to stay in some stable orbit is more as compared to its behavior while moving around Schwarzschild black hole. In Figure 5, different regions of effective potential which are linked to escape and bounded motion of the particle are shown. Here, $\alpha$ and $\beta$ are the regions which correspond to stable orbits for $b=0.5$. For $b=0$, there is only one stable region represented by $\gamma$ which is related to stable orbits. Dotted line represents the minimum energy required to escape from the vicinity of black hole. If the particle has energy $\mathscr{E} \geq 1$ and moves toward the black hole, it will bounce back to infinity which is represented by $\kappa$. In Figure 6, we are comparing the effective potentials of extremal black hole in the presence of magnetic field and without magnetic field. One can notice that, for $b=0.5$, the effective potential has two 


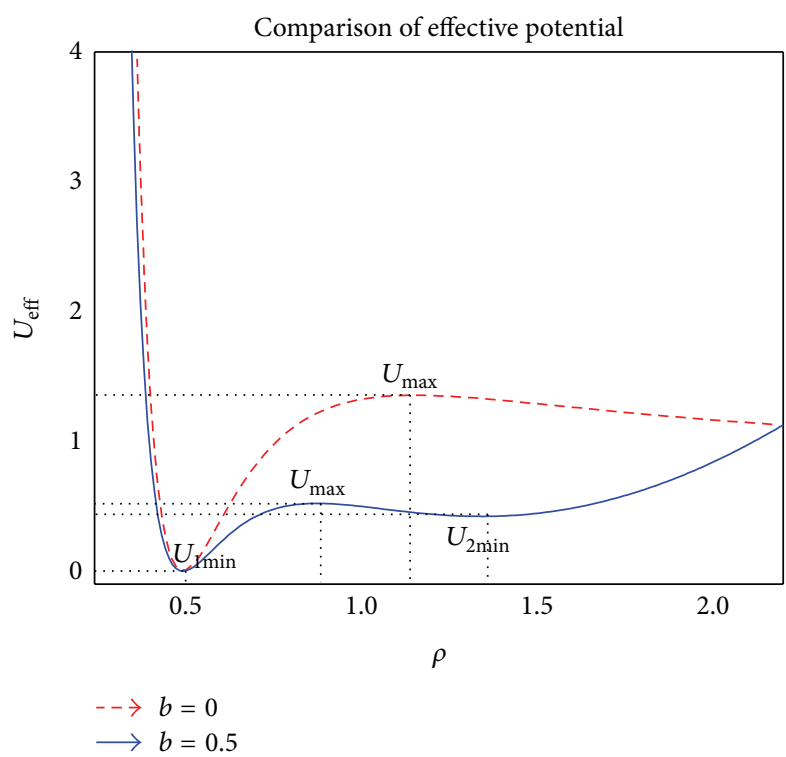

FIGURE 3: Effective potentials (with magnetic field, and, in its absence, a comparison) versus $\rho$.

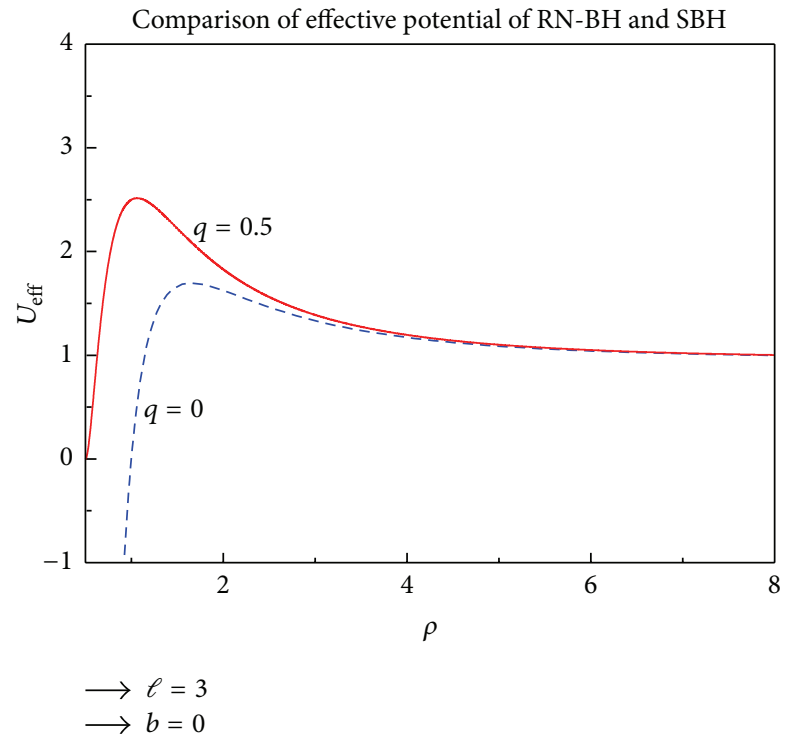

FIGURE 4: Effective potentials (34) as a function of $\rho$.

local minima which corresponds to two stable regions while for $b=0$ it has only one minimum. We use the notations $U_{\max }$ and $U_{\min }$ for unstable and stable circular orbits of the particle, respectively. Here, $U_{1 \mathrm{~min}}$ corresponds to ISCO which coincides with ISCO of the case when $b=0$ (dotted curve) and $U_{2 \min }$ correspond to stable circular orbits which occur due to presence of magnetic field. Therefore, we can say that magnetic field contributes to increasing the stability of the orbits. In Figure 7, we have plotted the magnetic field as a function of $\rho$. One can notice that magnetic field decreases abruptly as particle moves away from the source (black hole). We have plotted the angular momentum $\ell$ as a function of $\rho$ in Figure 8; it is clear that $\ell \rightarrow \infty$ for $\rho=0.5$. Angular

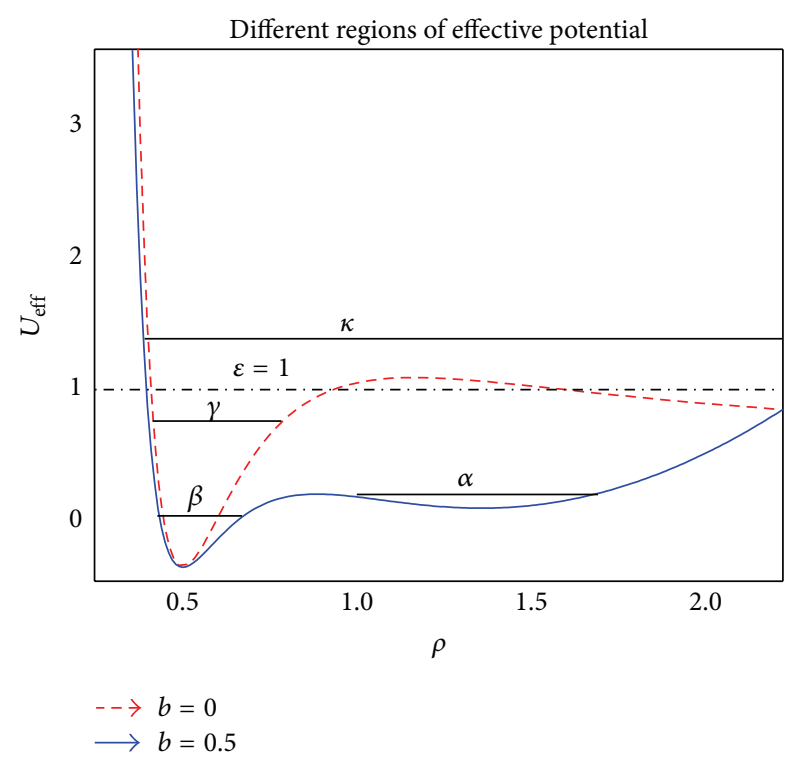

FIGURE 5: $\alpha$ and $\beta$ are the regions which correspond to stable orbits for $b=0.5$. For $b=0$, the region $\gamma$ corresponds to stable orbits.

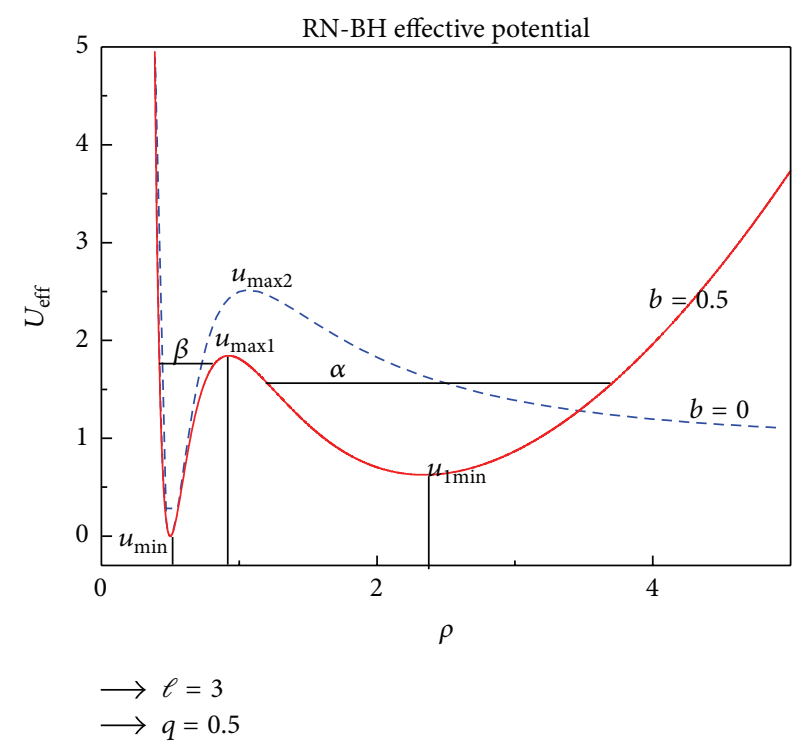

Figure 6: Behavior of effective potentials (34) with and without magnetic field versus $\rho$.

momentum $\ell_{ \pm}$for ISCO as a function of magnetic field $b$ is plotted in Figure 8.

\section{Trajectories of Escape Velocity}

Escape velocity of the charged particle is plotted in Figure 9; the shaded region corresponds to escape of particle from ISCO to $\infty$ and $-\infty$, respectively. The solid curve represents the minimum velocity which is required to escape from the ISCO. The unshaded region represents that if the value of velocity lies in this region then particle will remain in the ISCO or some other stable orbits. In Figure 10, we are 


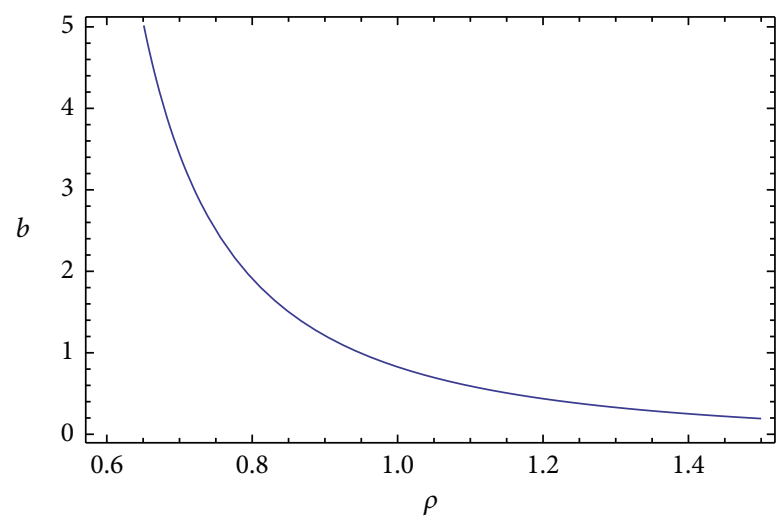

Figure 7: Magnetic field $b$ as a function of $\rho$. One can notice that magnetic field decreases abruptly away from the black hole.

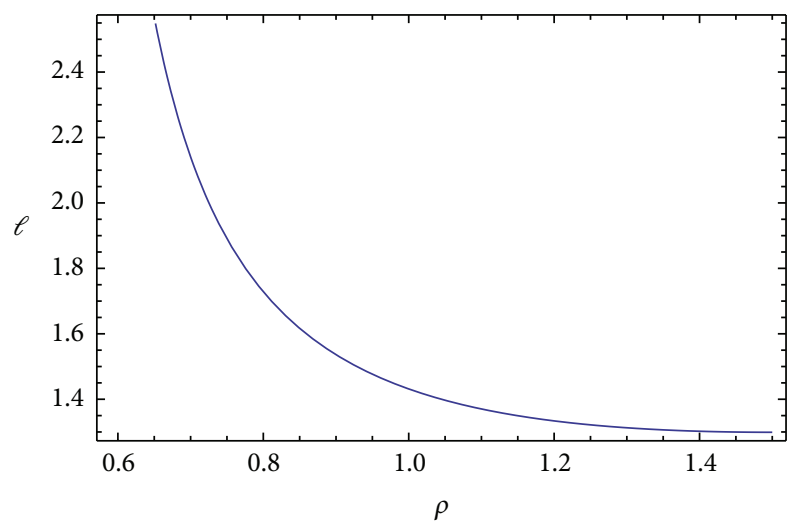

FIGURE 8: Angular momentum as a function of $\rho$.

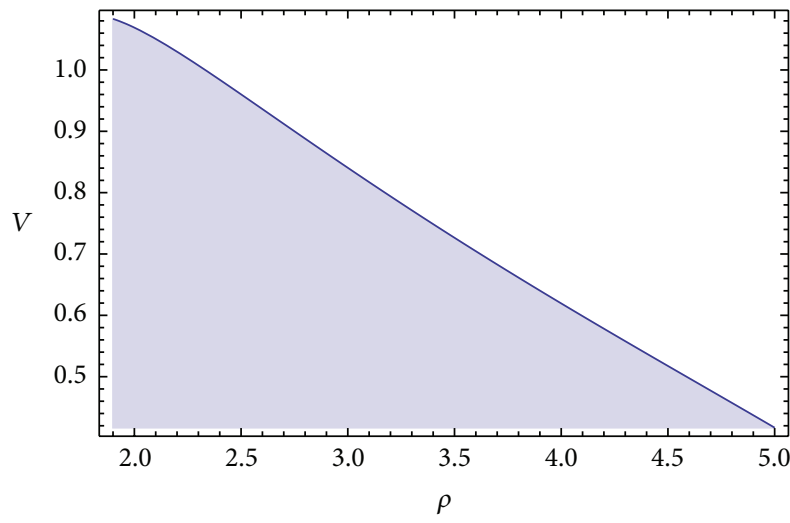

FIGURE 9: Solid curved line represents the minimum velocity required to escape from the ISCO. We have plotted the escape velocity $\left(v_{\text {esc }}\right)$ for $b=0.9$ and $\ell=1.5$.

comparing the escape velocity of a particle moving around the Schwarzschild black hole with the particle moving around the RN-BH. It is clear that the difference between the velocities is large near the black hole and it becomes almost the same as we move away from the black hole. Therefore, we can conclude that the effect of the charge of black hole on the motion of the particle is large and it is reducing as particle

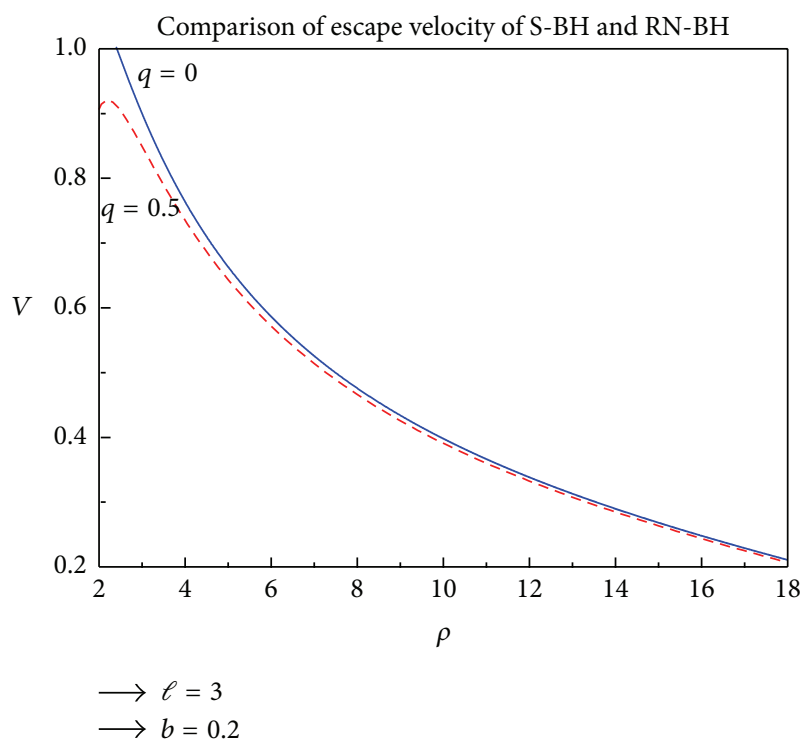

FIGURE 10: Escape velocity as function of $\rho$ for both Schwarzschild black hole and RN-BH.

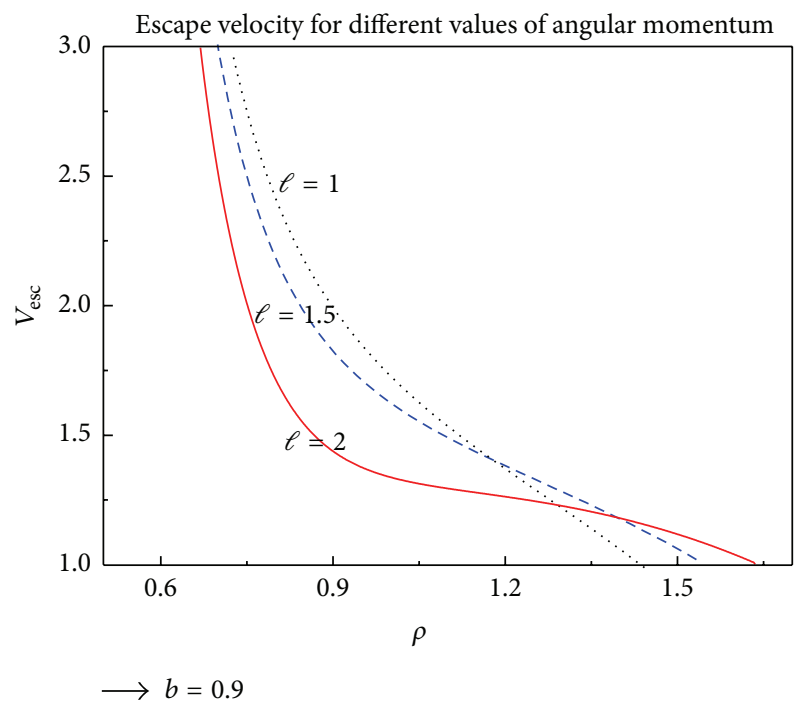

FIGURE 11: Escape velocity $\left(v_{\text {esc }}\right)$ against $\rho$ for different values of angular momentum $\ell$, for extremal $\mathrm{RN}-\mathrm{BH}$.

moves away from it. The behavior of escape velocity for extreme RN-BH is shown in Figure 11. The paths followed by the kicked (escaping) particle, moving initially in the ISCO, are shown in Figures 12, 13, and 14, which are obtained by solving (23), (24), and (25) numerically; we have taken initial radial velocity after collision as zero. We are interested to know the effect of magnetic field on the motion of charged particle (the magnitude of deformation produced in oscillatory motion). This effect increases as strength of magnetic field increases. Escape velocity is plotted in Figure 15 for different values of magnetic field $b$. Escape of the particle from the vicinity of black hole becomes easier in the presence of stronger magnetic field. As particle goes away from the black hole, its escape velocity becomes almost constant, just like 


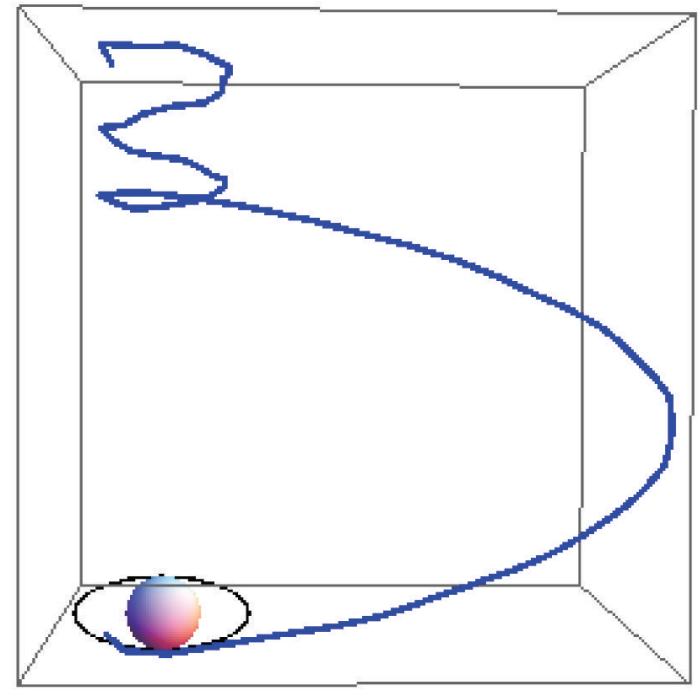

FIGURE 12: Trajectory of the escaping particle around RN-BH, for $b=1.5, \ell=1, \mathscr{E}=2, \rho[0]=1$.

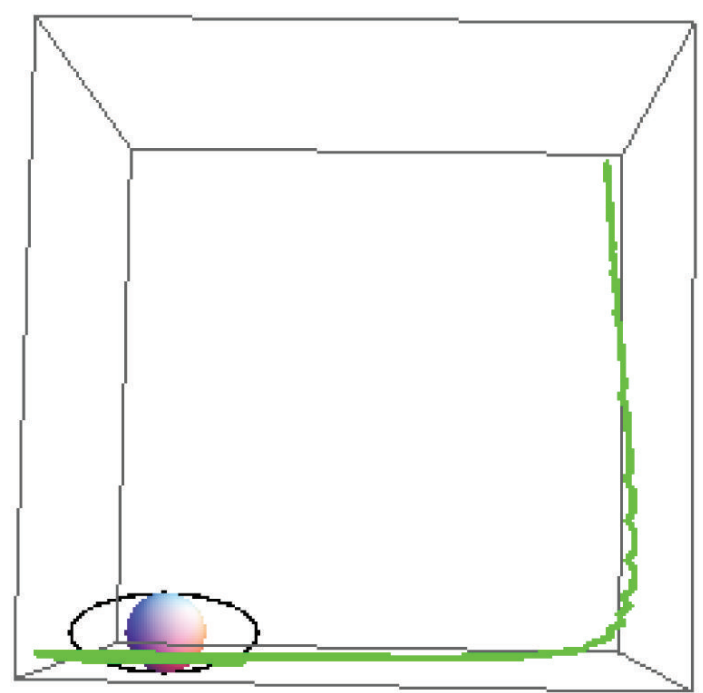

FIGURE 13: Trajectory of the escaping particle around RN-BH, for $b=1, \ell=1, \mathscr{E}=1, \rho[0]=1.2$.

the strength of magnetic field. Hence, presence of magnetic field will provide more energy to particle, so that it might easily escape from the vicinity of black hole. In Figure 16, connection of escape velocity with angular momentum is shown. It is clear that escape velocity of a particle with larger value of $\ell$ is greater compared to the particle with smaller value of $\ell$.

\section{Summary and Conclusion}

Motion of particles in the $\mathrm{RN}$ geometry in the presence of magnetic field is investigated in this paper. To avoid complications in the analysis, some assumptions are made, as mentioned in Section 2. We first studied the neutral particle moving around $\mathrm{RN}-\mathrm{BH}$ and derived the expressions for the

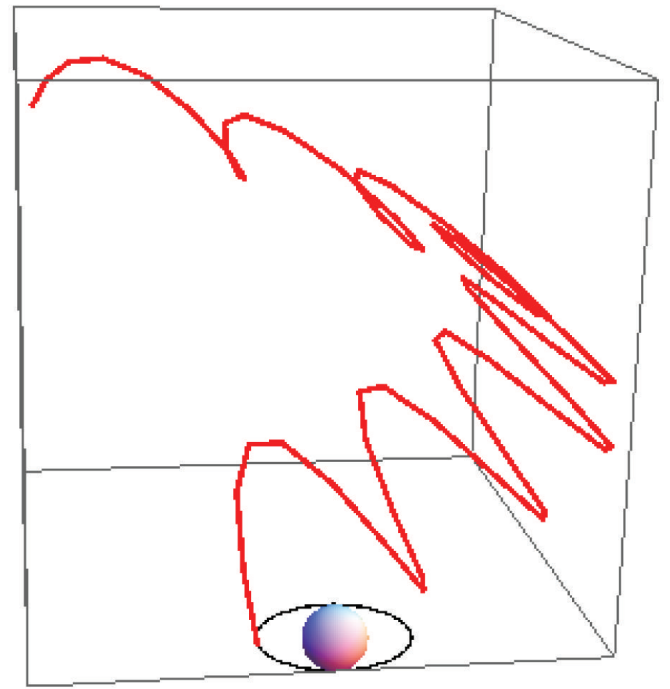

FIgURE 14: Trajectory of the escaping particle around RN-BH, for $b=0.5, \ell=1, \mathscr{E}=1, \rho[0]=0.51$.

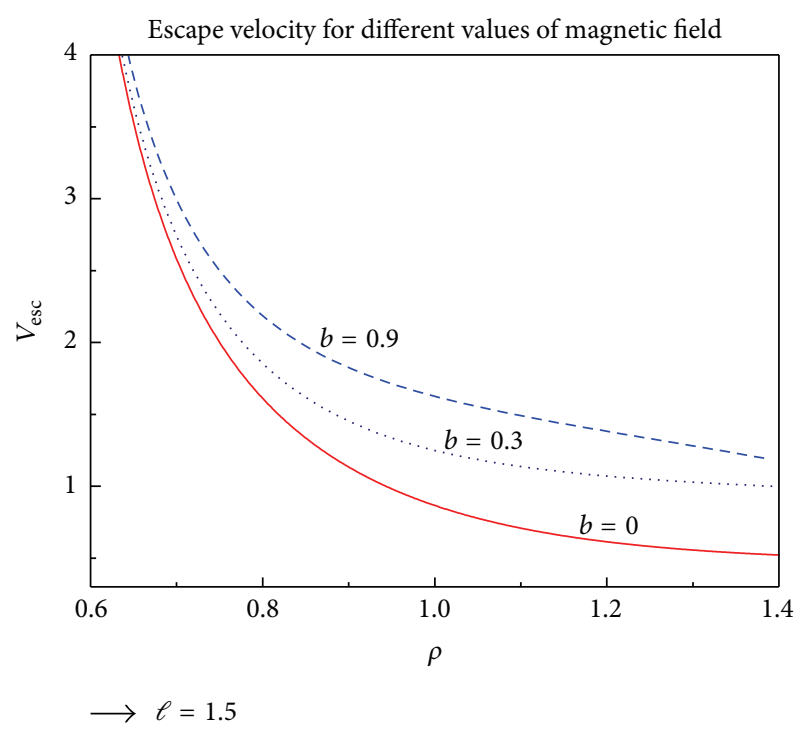

FIGURE 15: Effect of magnetic field $b$ on escape velocity $\left(v_{\text {esc }}\right)$.

energy and azimuthal angular momentum of the particle corresponding to ISCO. We obtained the expressions for escape velocity of the particle, after its collision with some other particles. Then, analysis for a charged particle is done, and dynamical equations of $\theta$ and $r$ are obtained. Effect of angular momentum and magnetic field on motion of neutral and charged particles is observed graphically. It is noticed that a particle with less angular momentum will approach the black hole more closely, as compared to the case when angular momentum is large. We find out the condition on energy of the particle required to escape or to remain bounded in orbit. Expressions for escape velocity of a charged particle moving around $\mathrm{RN}-\mathrm{BH}$, in the presence of magnetic field in the vicinity of black hole, are also obtained. Trajectories of the escaping charged particle are also shown graphically. It is observed that 


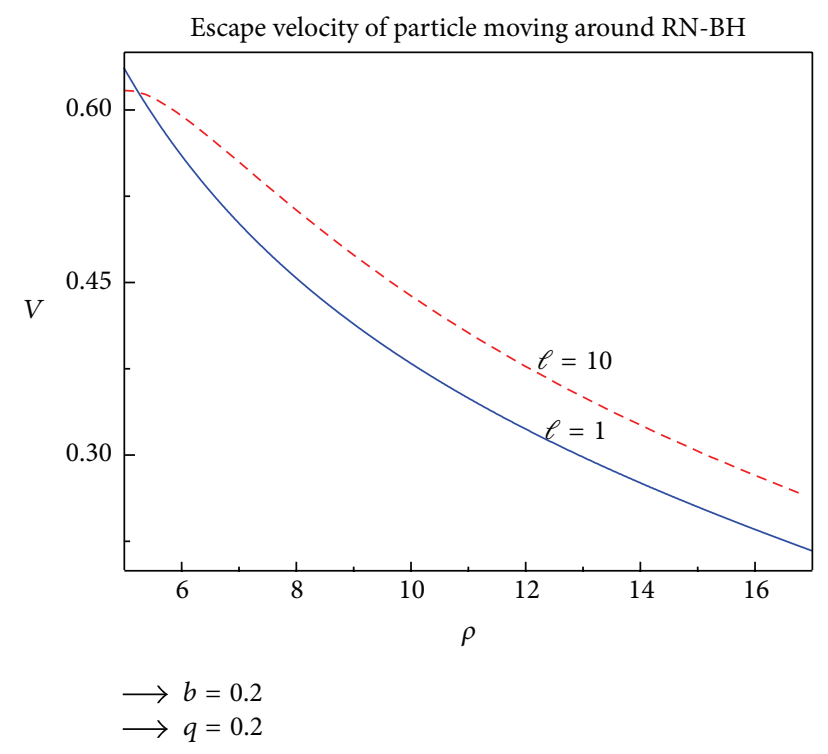

FIGURE 16: Escape velocity $\left(v_{\mathrm{esc}}\right)$ against $\rho$ for different values of angular momentum $\ell$.

a slight change in the initial condition of the colliding particle affects the escaping behavior. Presence of magnetic field also disturbs the escaping trajectories. Behavior of effective potentials is studied in detail and its effect on the stability of the orbits is explained graphically. More importantly, a comparison of effective potentials, obtained in the presence and absence of magnetic field, is established. It is seen that presence of magnetic field increases the stability of the orbits of the moving particles; in fact, two stable regions (local minima) are obtained in contrast to the only stable region obtained in the case when magnetic field is absent. Such analysis helps us to understand the effect of black hole on its surrounding matter. We intend to extend the similar analysis for $\mathrm{RN}$-de-Sitter black hole.

\section{Conflict of Interests}

The authors declare that there is no conflict of interests regarding the publication of this paper.

\section{References}

[1] V. P. Frolov and I. D. Novikov, Black Hole Physics, Basic Concepts and New Developments, Springer, 1998.

[2] N. A. Sharp, "Geodesics in black hole space-times," General Relativity and Gravitation, vol. 10, no. 8, pp. 659-670, 1979.

[3] M. A. Podurets, "Asymptotic behavior of the optical luminosity of a star in gravitational collapse," Astronomicheskii Zhurnal, vol. 41, p. 1090, 1964.

[4] W. L. Ames and K. S. Throne, "The optical appearance of a star that is collapsing through its gravitational radius," The Astrophysical Journal, vol. 151, pp. 659-670, 1968.

[5] C. V. Borm and M. Spaans, "The influence of magnetic fields, turbulence, and UV radiation on the formation of supermassive black holes," Astronomy \& Astrophysics, vol. 553, article L9, 4 pages, 2013.

[6] J. C. Mckinney and R. Narayan, "Disc-jet coupling in black hole accretion systems-II. Force-free electrodynamical models," Monthly Notices of the Royal Astronomical Society, vol. 375, no. 2, pp. 531-547, 2007.

[7] P. B. Dobbie, Z. Kuncic, G. V. Bicknell, and R. Salmeron, "New global 3D MHD simulations of black hole disk accretion and outflows," Proceedings of the International Astronomical Union, vol. 4, Symposium S259, pp. 129-130, 2008.

[8] R. Znajek, "On being close to a black hole without falling in," Nature, vol. 262, no. 5566, pp. 270-271, 1976.

[9] R. D. Blandford and R. L. Znajek, "Electromagnetic extraction of energy from Kerr black holes," Monthly Notices of the Royal Astronomical Society, vol. 179, no. 3, pp. 433-456, 1977.

[10] V. P. Frolov, "Weakly magnetized black holes as particle accelerators," Physical Review D, vol. 85, no. 2, Article ID 024020, 2012.

[11] S. Koide, K. Shibata, T. Kudoh, and D. L. Meier, "Extraction of black hole rotational energy by a magnetic field and the formation of relativistic jets," Science, vol. 295, no. 5560, pp. 16881691, 2002.

[12] K. N. Mishra and D. K. Chakraborty, "Orbits of a charged particle in a skew uniform magnetic field on kerr back ground geometry," Astrophysics and Space Science, vol. 260, no. 4, pp. 441-454, 1998.

[13] E. Teo, "Spherical photon orbits around a Kerr black hole," General Relativity and Gravitation, vol. 35, no. 11, pp. 1909-1926, 2003.

[14] S. Hussain, I. Hussain, and M. Jamil, "Dynamics of a charged particle around a slowly rotating Kerr black hole immersed in magnetic field," The European Physical Journal C, vol. 74, no. 12, article 3210, 2014.

[15] M. Jamil, S. Hussain, and B. Majeed, "Dynamics of particles around a Schwarzschild-like black hole in the presence of quintessence and magnetic field," The European Physical Journal C, vol. 75, article 24, 2015.

[16] S. Hussain and M. Jamil, "Timelike geodesics of a modified gravity black hole immersed in an axially symmetric magnetic field," Physical Review D, vol. 92, no. 4, Article ID 043008, 2015.

[17] G. Z. Babar, M. Jamil, and Y.-K. Lim, "Dynamics of a charged particle around a weakly magnetized naked singularity," International Journal of Modern Physics D, vol. 25, no. 2, Article ID 1650024, 2016.

[18] R. Kaya, "Motion of charged particles in a spacetime of a five dimensional rotating black hole in a magnetic field," General Relativity and Gravitation, vol. 39, no. 2, pp. 211-221, 2007.

[19] O. B. Zaslavskii, "Acceleration of particles by nonrotating charged black holes," JETP Letters, vol. 92, no. 9, pp. 571-574, 2011.

[20] D. Pugliese, H. Quevedo, and R. Ruffini, "Motion of charged test particles in Reissner-Nordström spacetime," Physical Review D, vol. 83, no. 10, Article ID 104052, 2011.

[21] A. M. Al Zahrani, V. P. Frolov, and A. A. Shoom, "Critical escape velocity for a charged particle moving around a weakly magnetized Schwarzschild black hole," Physical Review D, vol. 87, no. 8, Article ID 084043, 2013.

[22] S. Chandrasekhar, The Mathematical Theory of Black Holes, Oxford University Press, Oxford, UK, 1983. 
[23] V. P. Frolov and A. A. Shoom, "Motion of charged particles near a weakly magnetized Schwarzschild black hole," Physical Review D, vol. 82, no. 8, Article ID 084034, 2010.

[24] R. M. Wald, "Black hole in a uniform magnetic field," Physical Review D, vol. 10, no. 6, pp. 1680-1685, 1974.

[25] A. N. Aliev and N. Ozdemir, "Motion of charged particles around a rotating black hole in a magnetic field," Monthly Notices of the Royal Astronomical Society, vol. 336, no. 1, pp. 241248,1978 . 

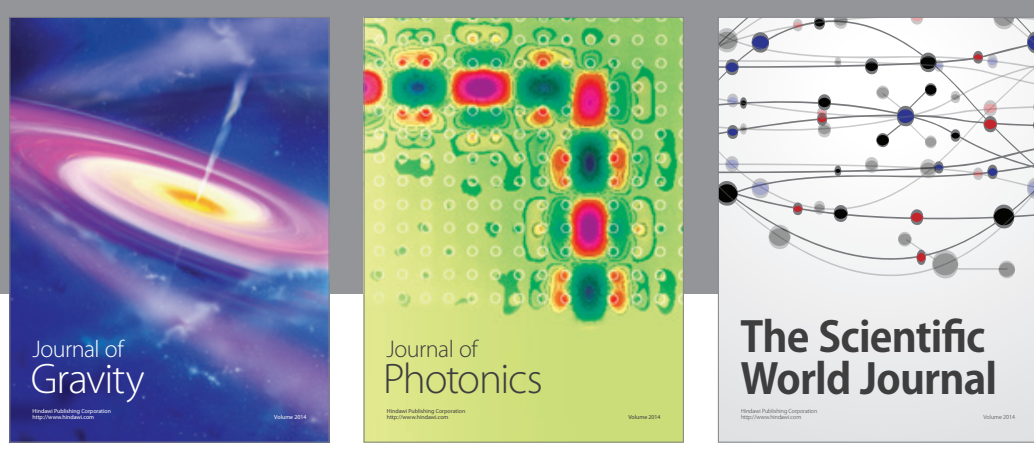

The Scientific World Journal
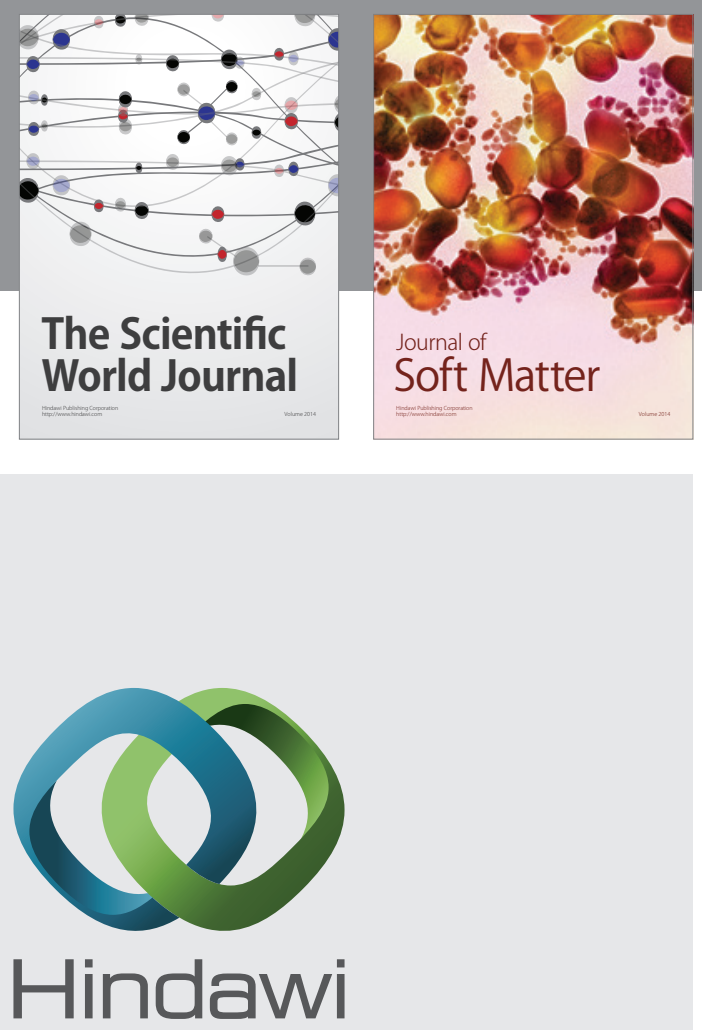

Submit your manuscripts at

http://www.hindawi.com

nternational Journal of

Statistical Mechanics
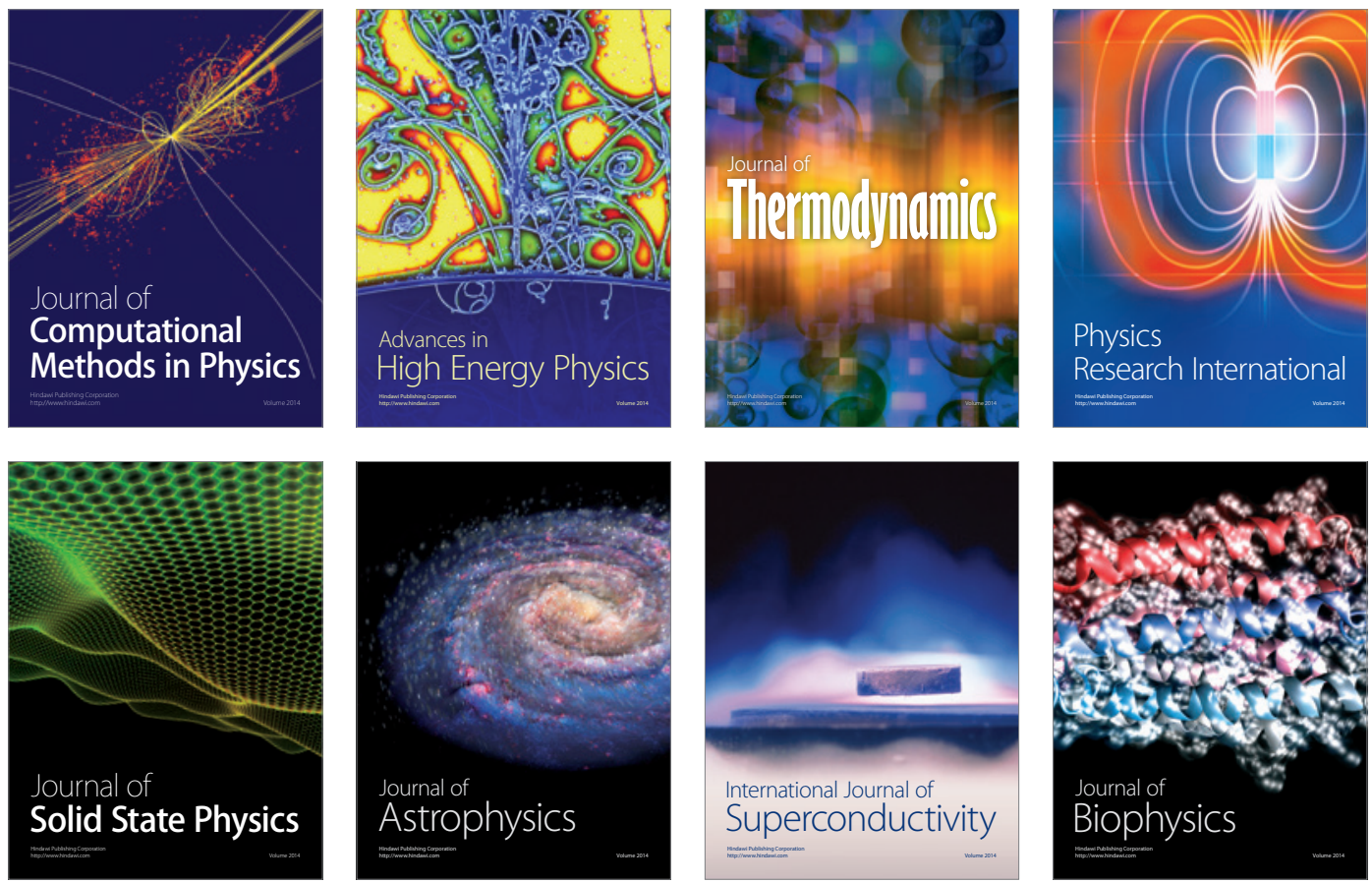
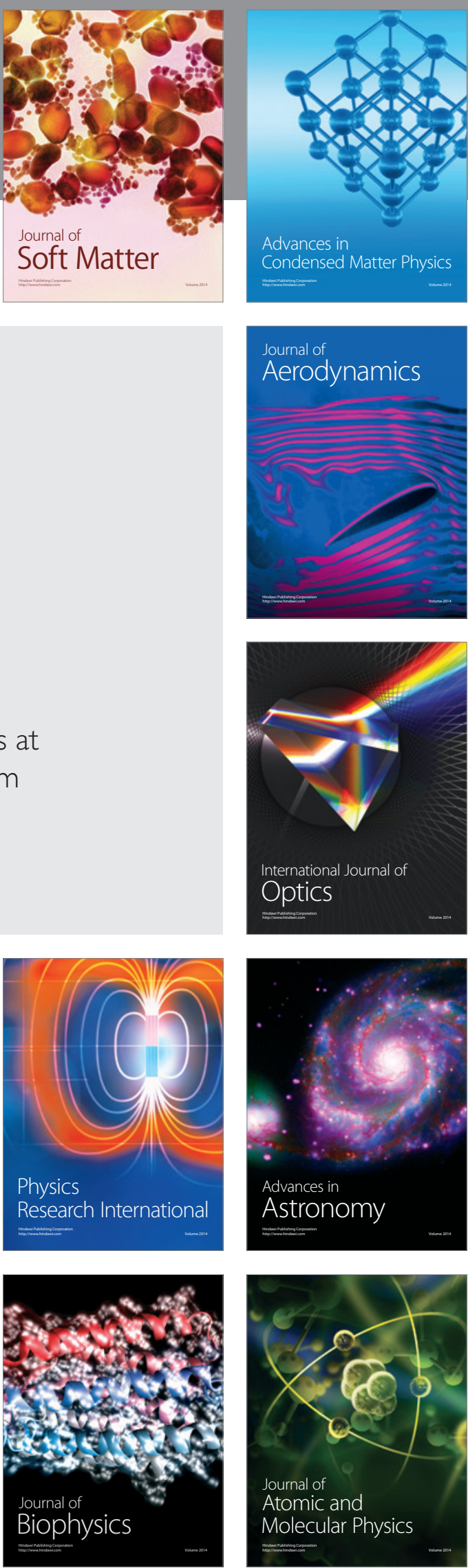